\title{
Etude de la qualité physico-chimique de l'eau de boisson dans deux localités du Bénin : Cotonou et Dassa-Zoumè
}

\author{
Virginie GBOHAIDA ${ }^{1}$, D. C. Pascal AGBANGNAN ${ }^{1 *}$, Mafuyu Bucku NGOSSANGA ${ }^{1}$, \\ S. Espérance MEDOATINSA ${ }^{1}$, Léonce F. C. DOVONON ${ }^{1,2}$, D. Valentin WOTTO ${ }^{3}$, \\ Félicien AVLESSI ${ }^{1}$ et Dominique C. K. SOHOUNHLOUE ${ }^{1}$

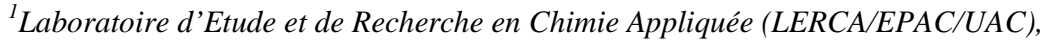 \\ 01 BP2009 Cotonou, Bénin. \\ ${ }^{2}$ Laboratoire d'Analyse des Eaux de la Direction Générale de l'Eau (DG-Eau), \\ 01 BP 385 Cotonou, Bénin. \\ ${ }^{3}$ Laboratoire de Chimie Physique (LCP/FAST/CHIMIE/UAC) 01 BP526 Cotonou, Bénin. \\ *Auteur correspondant ; E-mail: pascal.agbangnan@uac.bj, cokou2005@yahoo.fr
}

\section{RESUME}

L'eau, source potentielle de vie, doit subir diverses analyses physico-chimiques qui définiront sa qualité pour la consommation humaine afin d'éviter les risques de maladies hydriques pour les consommateurs. La présente étude vise à déterminer la qualité physico-chimique de l'eau de boisson des populations de deux villes du Bénin : Dassa-Zoumè et Cotonou. Trois points de prélèvement ont été identifiés dans chacune des deux localités pour un total de six (06) échantillons. Dix-neuf paramètres physico-chimiques ont été évalués sur ces échantillons. Certains paramètres (température, $\mathrm{pH}$, turbidité et conductivité) susceptibles de modification au cours du transport ont été déterminés "in situ". Les résultats obtenus ont montré que l'eau des deux localités que consomment les populations est de bonne qualité sur le plan physico-chimique. Néanmoins, l'eau de consommation des populations de Dassa est plus alcaline et plus dure que celle de Cotonou: soit respectivement de 390 à $480 \mathrm{mg} / \mathrm{l}$ et de 25 à $35 \mathrm{mg} / \mathrm{l}$ puis de 232 à $260 \mathrm{mg} / \mathrm{l}$ et de 15 à $27 \mathrm{mg} / \mathrm{l}$. Ce constat pourrait expliquer le caractère moins moussant et le goût terne souvent remarqués au niveau de l'eau de Dassa et serait lié à la nature géologique des sols traversés.

(C) 2016 International Formulae Group. All rights reserved

Mots clés : Qualité physico-chimique, eau de boisson, Bénin.

\section{Study of the physico-chemical quality of drinking water in two localities of Benin: Cotonou and Dassa-Zoumè}

\begin{abstract}
Water, potential source of life, must undergo various physico-chemical analyzes that will define its quality for human consumption in order to avoid the risk of water-borne diseases to consumers. The present study aims to determine the physico-chemical quality of population's drinking water in two cities of Benin: Cotonou and Dassa-Zoume. Three sampling points were identified in each of the two localities for a total of six (06) samples. Nineteen physico-chemical parameters were evaluated on these samples. Some parameters (temperature, $\mathrm{pH}$, turbidity and conductivity) which may be modified during transport were determined "in
\end{abstract}


situ". The results showed that the water consumed by the populations of both localities has a good physicochemical quality. Nevertheless, drinking water of Dassa's populations was more alkaline and harder than that of Cotonou: respectively 390-480 mg/l and 232-260 mg/l for Dassa and then $25-35 \mathrm{mg} / \mathrm{l}$ and $15-17 \mathrm{mg} / \mathrm{l}$ for Cotonou. This finding may explain the less foaming character and dull taste often noticed in Dassa's water and would be linked to the geological nature of the soils traversed.

(C) 2016 International Formulae Group. All rights reserved

Keywords: Physico-chemical quality, drinking water, Benin.

\section{INTRODUCTION}

L'eau constitue un bien considéré, à l'instar de l'air, comme essentiel à la vie humaine. Elle est l'un des moteurs de l'organisation et du développement des territoires. Inégalement répartie sur la terre et présente en quantités parfois limitées, elle constitue un enjeu environnemental majeur (Dovonon, 2011). Avoir l'eau à disposition en quantité suffisante et en qualité, contribue au maintien de la santé (Makoutode et al., 1999). La quantité et la qualité des ressources en eau disponibles posent actuellement des problèmes de plus en plus complexes et difficiles à résoudre notamment dans les pays sous-développés (Sintondji et al., 2008). L'environnement des humains dépend de l'eau qui, à son tour, dépend de la dynamique environnementale globale et locale. De ce fait, en recherche, comme chez les décideurs, l'eau et l'environnement sont souvent associés (Sal, 2010).

Le degré de qualité exigible des eaux dépend évidemment de leurs usages, et surtout la qualité de l'Eau Destinée à la Consommation Humaine (EDCH), qui, ellemême dépend des ressources en eau disponibles (Festy et al., 2003). Pour consommer l'eau sans danger, une politique de maîtrise et de gestion doit prendre en compte les caractéristiques de l'eau, molécule précieuse. L'eau doit donc subir diverses analyses physico-chimiques qui définiront sa qualité, pour la consommation humaine, afin d'éviter les risques de maladies hydriques pour les consommateurs.

A Cotonou, comme à Dassa, les eaux fournies par la Société Nationale des Eaux du
Bénin (SONEB) proviennent des forages (DG-Eau, 2007). Ces eaux subissent un ensemble de traitement avant d'être distribuées à la population. L'efficacité de ces traitements dépend non seulement des réactifs qui interviennent dans le processus, mais aussi de la dose appliquée conformément à la norme maximale admise. Certains consommateurs, surpris de voir changer leur eau du point de vue organoleptique (couleur et turbidité), se demandent si l'eau que leur fournit la SONEB est vraiment de bonne qualité (Agbataou, 2010). C'est dans cet ordre d'idée que cette étude se propose d'évaluer la qualité physicochimique de l'eau de consommation distribuée par la SONEB dans les communes de Cotonou et de Dassa à travers l'évaluation de quelques paramètres physico-chimiques.

\section{MATERIEL ET METHODES \\ Zones d'étude}

Ce travail a été effectué au Laboratoire de la DG-Eau et au Laboratoire d'Etude et de Recherche en Chimie Appliquée de l'Ecole Polytechnique de l'Université d'AbomeyCalavi avec des échantillons d'eau prélevés dans trois quartiers de Cotonou (Mênontin, Agontikon et Jéricho) et de Dassa-Zoumè (Carrefour Y, Ayédéro et Essékpéré) pour un total de six (06) échantillons d'eau. La ville de Cotonou, objet de cette étude, est située dans le département de l'Atlantique au Sud du Bénin entre $6^{\circ} 20^{\prime}$ et $6^{\circ} 23^{\prime}$ de latitude Nord et $2^{\circ} 22^{\prime}$ et $2^{\circ} 30^{\prime}$ de longitude Est (Odoulami, 2009). Elle se situe dans la plaine côtière, possède des sols sableux qui sont généralement pauvres en matières organiques avec une faible capacité d'échange et un faible 
pouvoir de rétention en eau. Cette ville est caractérisée par un climat sub-équatorial avec une alternance de deux saisons pluvieuses (Avril à Juillet et Septembre à Novembre) et de deux saisons sèches (Akomagni, 2006). La pluviométrie à Cotonou varie entre 900 et $1200 \mathrm{~mm}$ et la température moyenne est de $27{ }^{\circ} \mathrm{C}$ environ. Dassa-Zoumè est une ville située dans le département des Collines à environ $203 \mathrm{~km}$ de Cotonou (Dovonon, 2011). Ce département est compris entre $7^{\circ}$ et $8^{\circ}$ de latitude Nord et les méridiens $1^{\circ} 60^{\prime}$ et $2^{\circ} 80^{\prime}$ de longitude Est. Il est situé dans la partie centrale de la République du Bénin, pays de l'Afrique de l'Ouest, dans la zone tropicale entre les parallèles $6^{\circ} 30^{\prime}$ et $12^{\circ} 30^{\prime}$ de latitude Nord et les méridiens $1^{\circ}$ et $3^{\circ} 40^{\prime}$ de longitude Est (Dovonon et al., 2011). Le climat à DassaZoumè, va du type équatorial à tropical de transition. On y compte une saison sèche et une saison pluvieuse. La moyenne pluviométrique est d'environ $1200 \mathrm{~mm} / \mathrm{an}$. Les températures connaissent des minima de $21{ }^{\circ} \mathrm{C}$ et des pointes à $36{ }^{\circ} \mathrm{C}$. L'évaporation est estimée à environ $1332 \mathrm{~mm} / \mathrm{an}$ (AwoAffouda, 2009). A Dassa, on note la présence des affleurements rocheux favorisant l'installation des sols minéraux bruts inaptes à l'agriculture.

\section{Echantillonnage et analyse de l'eau}

Le prélèvement d'un échantillon d'eau, en vue des analyses physico-chimiques est une opération qui nécessite beaucoup de soins. Il conditionne les résultats analytiques et reflète des conséquences sur l'interprétation qui en sera faite. Les échantillons ont été prélevés dans des bouteilles plastiques d'eau minérale de $1,5 \quad 1$ puis acheminés au laboratoire dans une glacière pour les analyses. Toutefois, le pH, la conductivité et la température ont été mesurés "in situ" grâce à un multimètre portatif (HANNA, HI 991300). Les bicarbonates, les chlorures, le calcium, le magnésium et la dureté totale ont été mesurés par titrimétrie tandis que l'ammonium, la couleur, le fer total, les iodures, les nitrates, les nitrites, les sulfates, les phosphates et les fluorures ont été déterminés par un spectrophotomètre de type HACH DR/2400 conformément aux méthodes d'analyse de l'Association Française de Normalisation, AFNOR (AFNOR, 1986 ; Rodier, 2009). Les analyses ont été réalisées en décembre 2014 juste après l'échantillonnage.

\section{RESULTATS \\ Paramètres physiques \\ Variation de la température de l'eau}

Les valeurs de température obtenues sont comprises entre $28{ }^{\circ} \mathrm{C}$ et $32{ }^{\circ} \mathrm{C}$. La Figure 1 traduisant la variation de la température de l'eau de boisson durant les trois moments (matin, midi et soir) de la journée dans les zones d'étude montre une légère augmentation de la température de l'eau à midi et une baisse au-delà. Ce qui montre que midi est le moment de forte température dans la journée. Notons que la température de l'eau de robinet varie très peu au cours de la journée.

\section{Couleur}

L'analyse de la couleur de l'eau de robinet de Cotonou et de Dassa (Figure 2) montre que l'eau de Agontikon à Cotonou est légèrement plus colorée que celles des autres quartiers avec 5 pour valeur de couleur, bien que n'atteignant pas la norme maximale requise qui est de $15 \mathrm{UC}$.

\section{Turbidité}

La norme établie sur la turbidité est de 5 Nephelometric Turbidity Unit (NTU). Les différents échantillons d'eau analysés ont présenté des valeurs de turbidité comprises entre 0 et 1 NTU, répondant à la norme. L'eau de Dassa n'a présenté aucune turbidité.

\section{Conductivité électrique}

Les valeurs de conductivité obtenues sont en dessous de la norme maximale (2000 $\mu \mathrm{S} / \mathrm{cm})$. Ces valeurs sont comprises entre 49 et $129 \mu \mathrm{S} / \mathrm{cm}$ à Cotonou puis entre 480 et 487 $\mu \mathrm{S} / \mathrm{cm}$ à Dassa (Figure 3). 
$p H$

Le $\mathrm{pH}$ de l'eau analysée est acide avec des valeurs comprises entre 5,4 et 6,8 (Figure 4). Les valeurs de $\mathrm{pH}$ obtenues à Cotonou ont été en dessous de la plage de $\mathrm{pH}$ admise qui est de 6,5 - 8,5 alors qu'à Dassa, les eaux analysées ont présenté des valeurs de $\mathrm{pH}$ se situant dans la marge.

\section{Paramètres chimiques \\ Composés minéraux azotés et phosphatés}

Les résultats obtenus dans le présent travail sur les composés minéraux azotés et phosphatés sont reportés dans le Tableau 1. La bonne qualité de ces eaux est confirmée par les résultats obtenus pour les composés minéraux azotés et phosphatés (Tableau 1), car il apparaît de très faibles quantités d'ions ammonium $\left(\mathrm{NH}_{4}{ }^{+}\right)$, nitrites $\left(\mathrm{NO}_{2}{ }^{-}\right)$, nitrates $\left(\mathrm{NO}_{3}{ }^{-}\right)$et phosphates $\left(\mathrm{PO}_{4}^{3-}\right)$.

\section{Bicarbonates}

Les résultats présentés sur la Figure 5 montrent que les ions bicarbonates sont en concentration très faible à Cotonou avec des valeurs allant de $15,25 \mathrm{mg} / \mathrm{l}$ à $21,35 \mathrm{mg} / \mathrm{l}$ et très élevée à Dassa $(237,9 \mathrm{mg} / \mathrm{l}$ à $292,8 \mathrm{mg} / \mathrm{l})$. On remarque que les concentrations en bicarbonates obtenues à Dassa sont plus de dix fois supérieures à celles de Cotonou.

\section{Magnésium}

$\mathrm{La}$ concentration maximale en magnésium admise est de $50 \mathrm{mg} / \mathrm{l}$. Les concentrations en magnésium obtenues variaient de 0,729 à $28,211 \mathrm{mg} / \mathrm{l}$ et semblent être négligeables dans l'eau de Cotonou (Figure 6).

\section{Calcium}

Le calcium est un métal alcalinoterreux extrêmement répandu dans la nature et en particulier dans les roches calcaires sous forme de carbonates. La concentration maximale admise est de $100 \mathrm{mg} / \mathrm{l}$. Les valeurs des analyses des échantillons des deux localités: Cotonou et Dassa, variaient de 4,008 à $60,120 \mathrm{mg} / \mathrm{l}$ (Figure 7). On observe que les concentrations dans la localité de Cotonou $(4,008$ à $5,611 \mathrm{mg} / \mathrm{l})$ sont très faibles devant celles de Dassa $(57,314$ à 60,120 $\mathrm{mg} / \mathrm{l})$. De l'analyse faite, il convient de dire que la concentration en calcium est négligeable dans l'eau de Cotonou, cependant la concentration en calcium dans l'eau de Dassa est plus proche de la norme.

\section{Dureté totale}

La dureté est basée sur la concentration des sels (calcium et magnésium) dissous contenus dans l'eau. Les valeurs de dureté totale dans la localité de Cotonou ont varié de 13 à $27 \mathrm{mg} / \mathrm{l}$ et celles de Dassa ont été très supérieures (232 à $260 \mathrm{mg} / \mathrm{l})$ à celles de Cotonou bien que répondant à la valeur limite qui est de $500 \mathrm{mg} / \mathrm{l}$ (Figure 8).

\section{Alcalinité}

La Figure 9 présente les valeurs d'alcalinité de l'eau de boisson échantillonnée à Cotonou et à Dassa. Au vu des résultats, la zone de Cotonou a présenté des teneurs allant de 25 à $35 \mathrm{mg} / \mathrm{l}$ et à Dassa, nous avons des teneurs très élevées (232 à $260 \mathrm{mg} / \mathrm{l})$.

\section{Iodures}

On a observé des concentrations d'iodures variant de 0,09 à $0,21 \mathrm{mg} / \mathrm{l}$, dont la plus élevée a été enregistrée à Dassa (Tableau $2)$.

Les résultats obtenus pour la quantification des autres ions tels que sulfates, fluorures, fer total et chlorures sont presque négligeables comparativement à la norme admise (Tableau 2). 


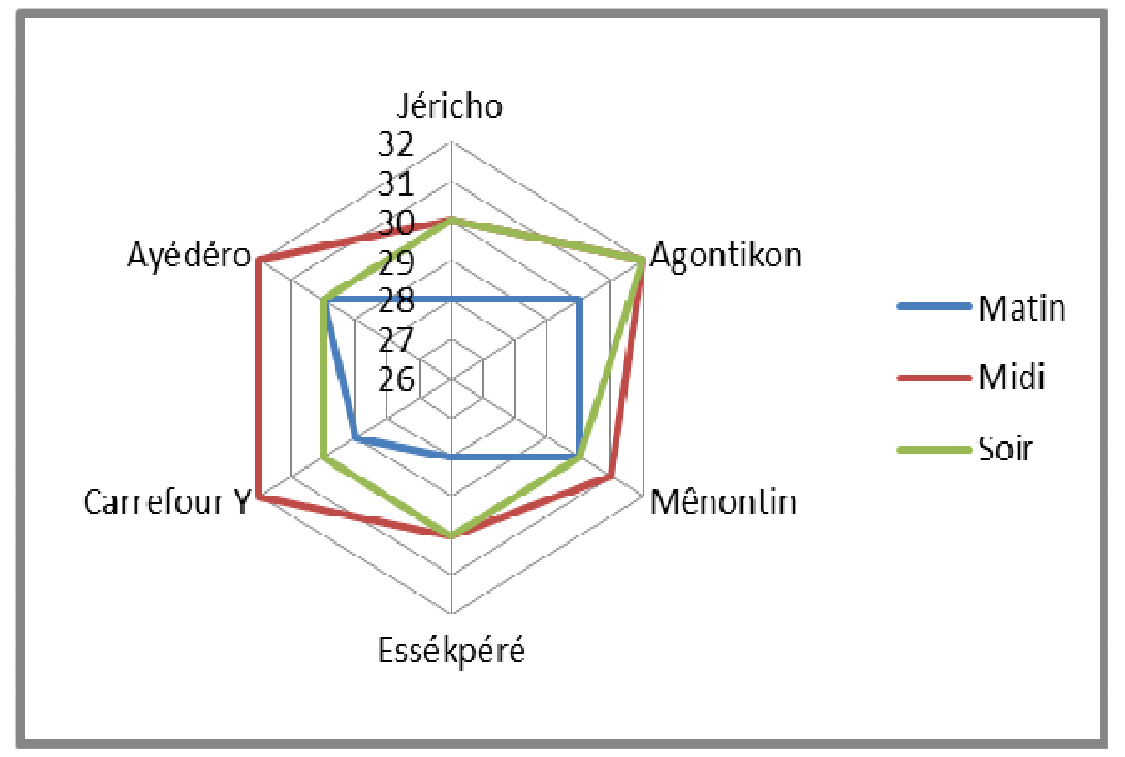

Figure 1: Variation de la température de l'eau de boisson de Cotonou et de Dassa dans la journée.

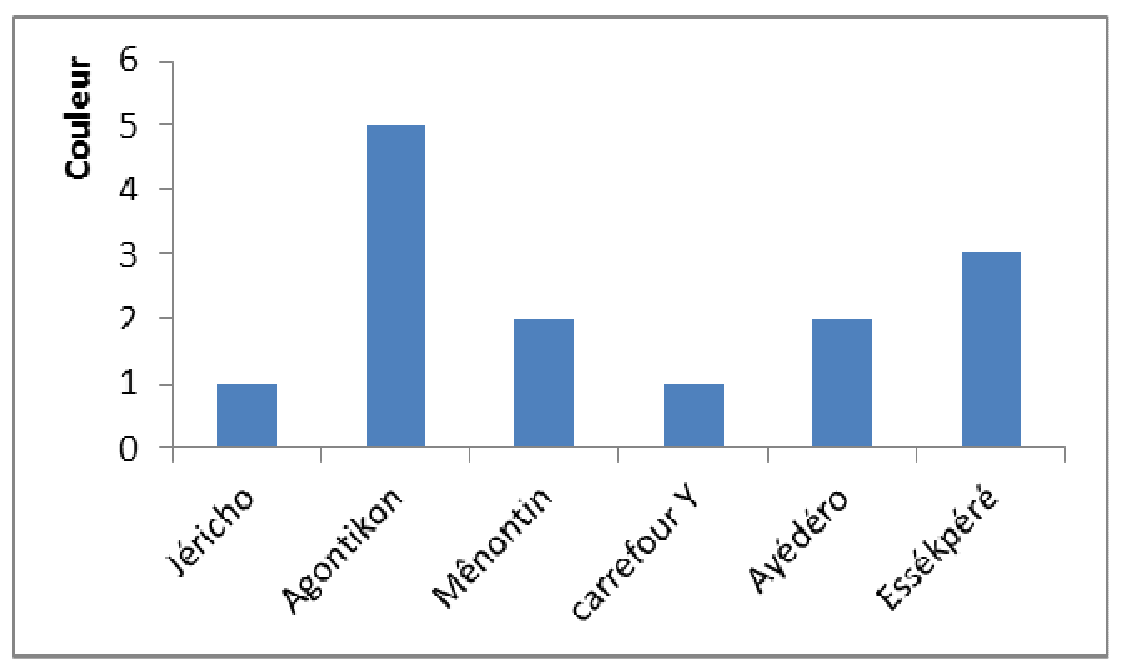

Figure 2: Quantification de la couleur des échantillons de Cotonou et de Dassa. 


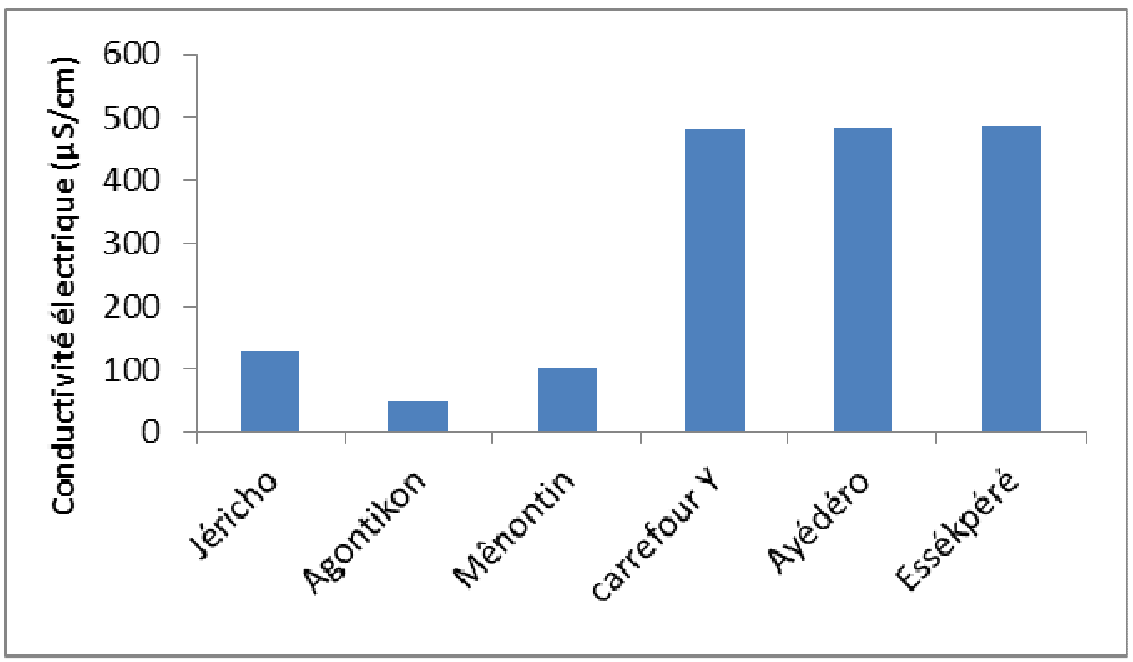

Figure 3: Conductivité des échantillons d'eau de Cotonou et de Dassa.

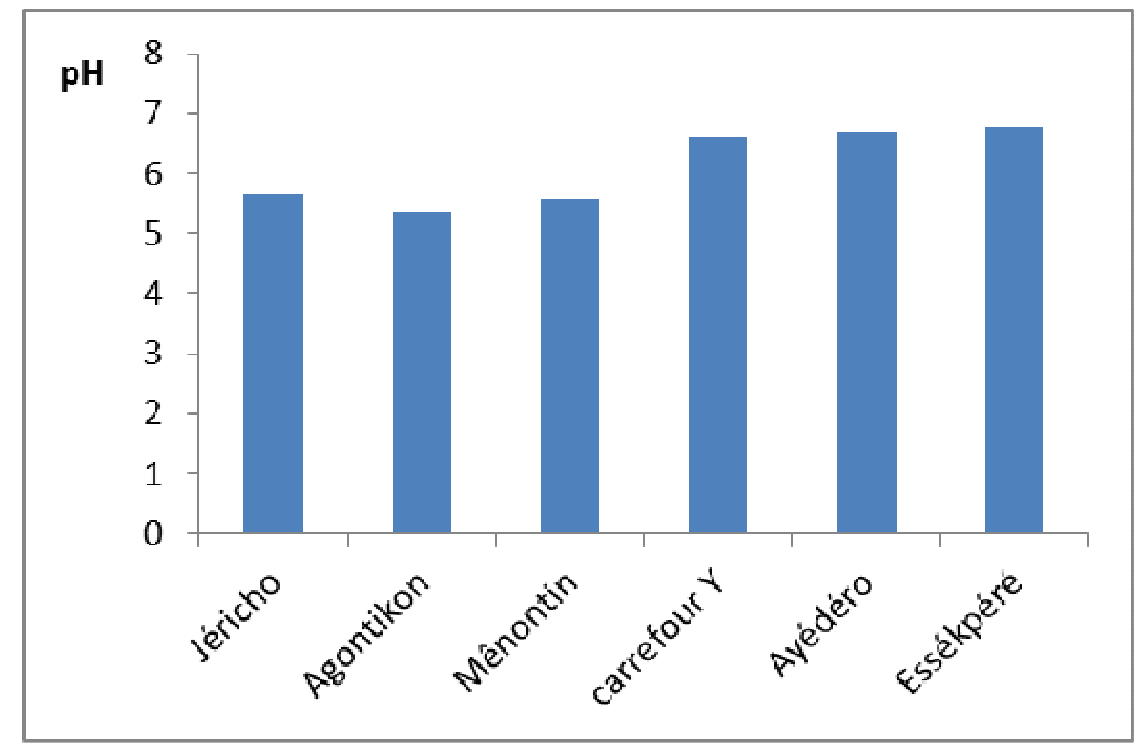

Figure 4: Répartition du pH des échantillons de Cotonou et de Dassa. 


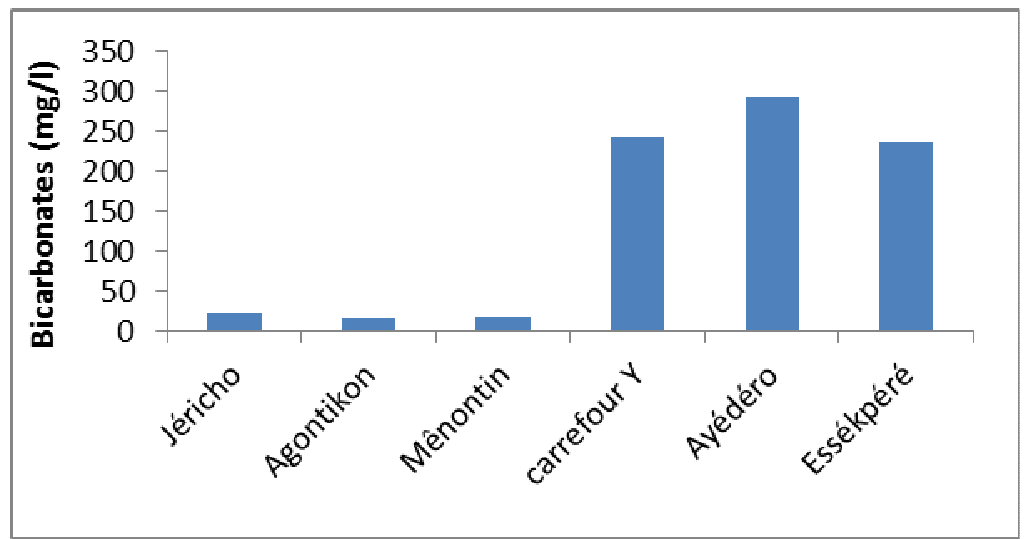

Figure 5: Concentration en bicarbonates des échantillons d'eau de Cotonou et de Dassa.

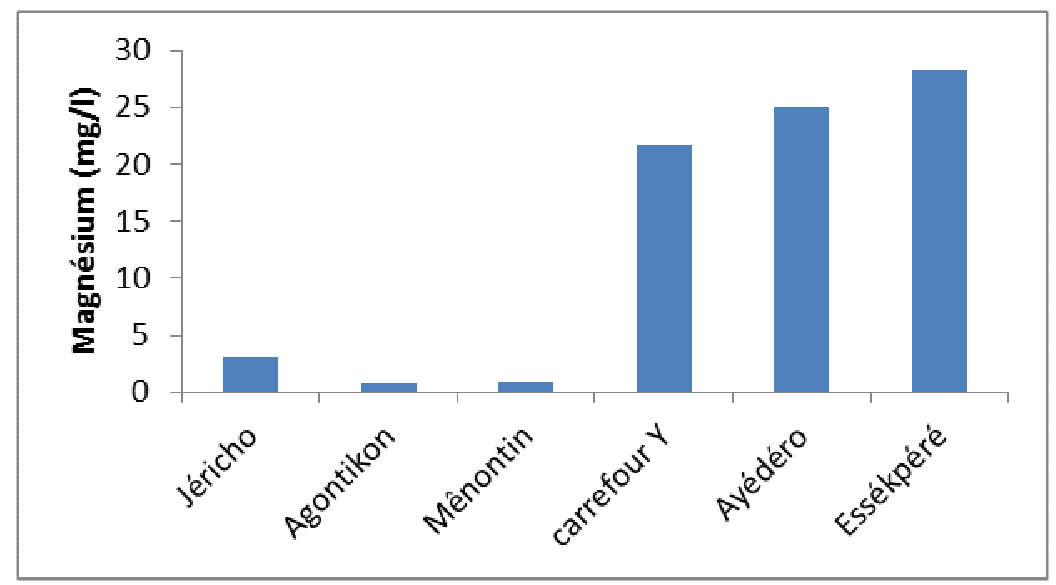

Figure 6: Concentration en magnésium des échantillons d'eau de Cotonou et de Dassa.

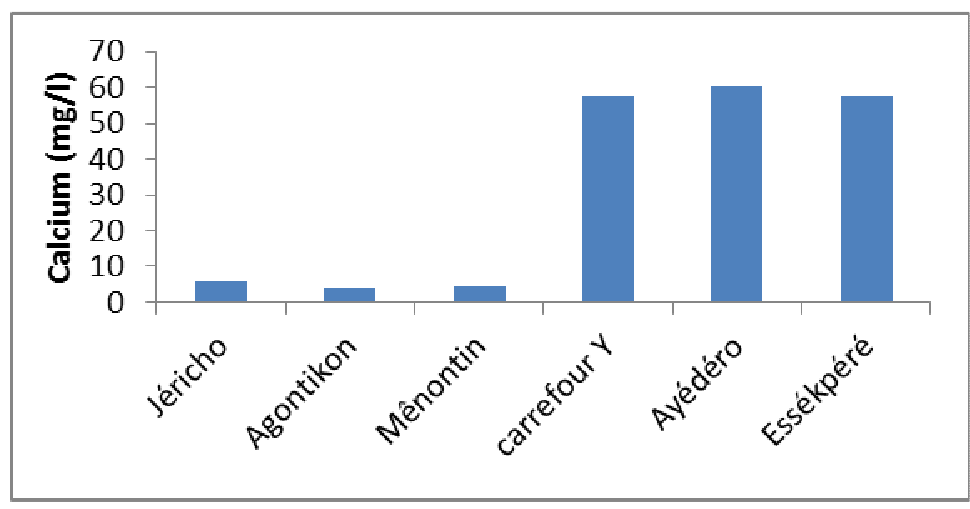

Figure 7: Concentration en calcium des échantillons d'eau de Cotonou et de Dassa. 


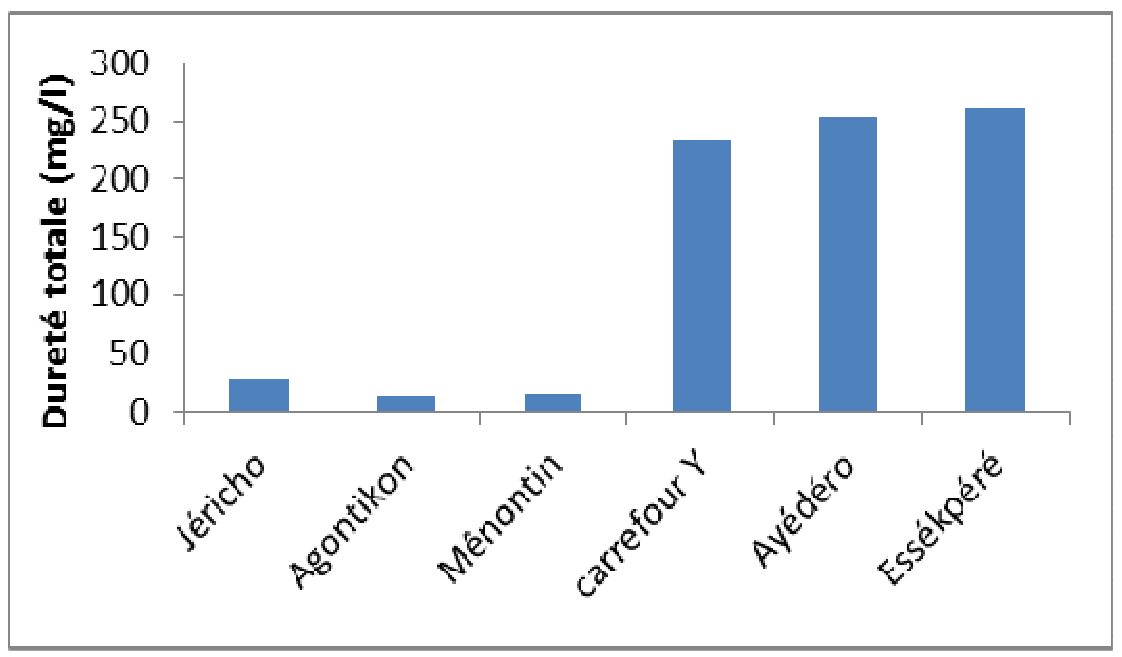

Figure 8: Dureté totale des échantillons d'eau de Cotonou et de Dassa.

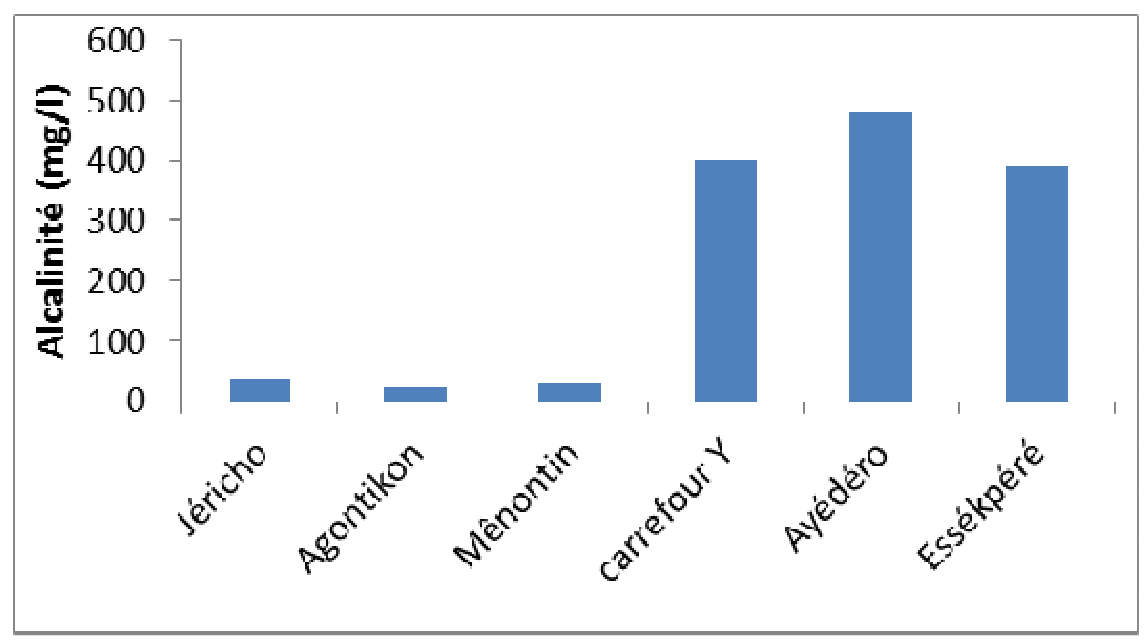

Figure 9: Alcalinité des échantillons d'eau de Cotonou et de Dassa. 
Tableau 1: Paramètres caractéristiques de l'azote et du phosphore minéraux des eaux.

\begin{tabular}{|c|c|c|c|c|c|c|c|}
\hline Paramètres & Mênontin & Agontikon & Jéricho & Carrefour Y & Ayédéro & Essékpéré & Norme au Bénin*** \\
\hline Ammonium $\mathrm{NH}_{4}^{+}(\mathrm{mg} / \mathrm{l})$ & 0,0387 & 0,0387 & 0,0387 & 0,1032 & 0,1032 & 0,1548 & 0,5 \\
\hline Nitrates $\mathrm{NO}_{3}^{-}(\mathrm{mg} / \mathrm{l})$ & 8,8 & 8,8 & 10,56 & 6,6 & 5,72 & 5,28 & 50 \\
\hline Nitrites $\mathrm{NO}_{2}^{-}(\mathrm{mg} / \mathrm{l})$ & 0,0099 & 0,0066 & 0,0066 & 0,0066 & 0,0066 & 0,0066 & 0,1 \\
\hline Phosphates $\mathrm{PO}_{4}^{3-}(\mathrm{mg} / \mathrm{l})$ & 1,74 & 2,01 & 1,93 & 2,31 & 1,57 & 0,31 & 5 \\
\hline
\end{tabular}

** Décret $\mathrm{N}^{\circ}$ 094-2001 du 20/02/2001 portant norme de l'eau potable en République du Bénin.

Tableau 2: Quelques paramètres physico-chimiques de l'eau de boisson de Cotonou et de Dassa-Zoumè.

\begin{tabular}{|c|c|c|c|c|c|c|c|}
\hline Paramètres & Mênontin & Agontikon & Jéricho & Carrefour Y & Ayédéro & Essékpéré & Norme au Bénin \\
\hline Sulfates $\mathrm{SO}_{4}{ }^{2-}(\mathrm{mg} / \mathrm{l})$ & 01 & 00 & 02 & 17 & 16 & 17 & 500 \\
\hline Fluorures $\mathrm{F}^{-}(\mathrm{mg} / \mathrm{l})$ & 0,0 & 0,08 & 0,23 & 0,54 & 0,89 & 0,62 & 1,5 \\
\hline Iodure $\mathrm{I}^{-}(\mathrm{mg} / \mathrm{l})$ & 0,09 & 0,16 & 0,17 & 0,18 & 0,21 & 0,18 & - \\
\hline Fer total $\mathrm{Fe}^{2+} / \mathrm{Fe}^{3+}(\mathrm{mg} / \mathrm{l})$ & 0,03 & 0,01 & 0,05 & 0,01 & 0,01 & 0,0 & 0,3 \\
\hline Chlorures $\mathrm{Cl}^{-}(\mathrm{mg} / \mathrm{l})$ & 21,3 & 26,625 & 46,15 & 28,4 & 33,73 & 23,08 & 250 \\
\hline
\end{tabular}




\section{DISCUSSION}

La variation de la température au cours de la journée n'a donc pas une influence significative sur la qualité de l'eau de robinet. Odoulami (2009) dans ses investigations sur les eaux de puits, de pluie et de la SONEB avait obtenu des résultats similaires. $\mathrm{La}$ température de l'eau de Cotonou et de Dassa a présenté la même tendance au cours de la journée, et le midi s'est révélé le moment le plus chaud de la journée dans les deux communes d'étude. La valeur de température de l'eau obtenue dans la matinée est similaire à celle obtenue par Dégbey et al. (2008) et Lagnika et al. (2014). En outre, les valeurs de température obtenues le matin dans cette étude sont similaires à celles obtenues par Adejuwon et Mbuk (2011) dans leurs investigations sur l'eau de puits. La température de l'eau dépend donc du moment de son prélèvement et est intimement liée à la température ambiante au moment du prélèvement.

Les valeurs de couleur obtenues sont inférieures à celles obtenues par Barhé et Bouaka (2013) dont les travaux sur l'eau de puits au Congo Brazzaville ont révélé des valeurs de couleur comprises entre 7 et 10.

Les valeurs de turbidité obtenues répondent bien à la norme requise et sont les mêmes que celles de Babadjide et al. (2011).

La conductivité électrique traduit le degré de minéralisation globale, elle renseigne sur le taux de salinité (Ould Cheikh et al., 2011). Les résultats de conductivité sont proches de ceux obtenus par Adejuwon et Mbuk (2011) sur l'eau de puits de Lagos au Nigéria avec des valeurs de conductivité comprises entre 22 et $315 \mu \mathrm{S} / \mathrm{cm}$. Cependant, ces résultats sont contraires à ceux de Tampo et al. (2014) qui ont obtenu des conductivités supérieures à la norme dans les eaux de puits de Lomé au Togo.

Les valeurs de $\mathrm{pH}$ sont similaires à celles obtenues par Lagnika et al. (2014) dans la commune de Pobé sur l'eau de puits. De même, ces valeurs de $\mathrm{pH}$ sont très proches de celles de Tampo et al. (2014) qui ont obtenu des valeurs inférieures à 6,5 dans les eaux des puits des quartiers périurbains de Lomé. Aussi, les valeurs de $\mathrm{pH}$ obtenues sont conformes aux résultats obtenus par Mahamane et Guel (2015) sur les eaux souterraines en Côte d'Ivoire. Elles sont par ailleurs différentes de celles de Derwich et al. (2010) qui ont obtenu des valeurs variant entre 7,1 et 8,1 dans les eaux des nappes alluviales du haut Sebou. Cette différence serait due à la nature très différente des roches, réservoirs naturels des eaux souterraines.

Les teneurs en composés minéraux azotés et phosphatés sont toutes inférieures aux valeurs limites recommandées par l'Organisation Mondiale de la Santé (OMS, 1994). En effet, l'ingestion des nitrates est un facteur de risque potentiel avéré pour la santé. Ils sont réduits en nitrites, qui se fixent sur l'hémoglobine à la place de l'oxygène et provoquent des difficultés respiratoires (asphyxie) : c'est la méthémoglobinémie (cyanose) qui touche essentiellement les nourrissons (nés ou en gestation) et présente un risque à court terme. Chez l'adulte, les nitrates seraient transformés en nitrites au niveau de la cavité buccale, puis en nitrosamines et autres composés nitrosés au niveau de l'estomac (Öztürk et Bektaş, 2004). Les résultats obtenus dans le présent travail sur les composés minéraux azotés et phosphatés sont similaires à ceux de Tampo et al. (2014) sur l'eau de forage mais contraires aux résultats de ces mêmes auteurs sur l'eau de puits. La forte concentration de ces minéraux dans les puits pourrait s'expliquer par le lessivage des fertilisants agricoles qui s'infiltrent dans ces puits très peu profonds situés à proximité des champs de maïs et de légumes.

Les teneurs en magnésium obtenues sont presque similaires à celles obtenues par Dovonon (2011) à Dassa dans ses investigations sur l'impact du fluorure dans les eaux souterraines.

Les résultats obtenus pour la quantification du calcium sont différents de ceux de Lagnika et al. (2014) et de Barhé et Bouaka (2013). Leurs études ont été menées sur les eaux de puits respectivement dans les 
zones de Pobè et Kétou au Bénin et au Congo Brazzaville. Cette différence serait due aux conditions géomorphologiques des zones d'étude. La zone de Dassa a la particularité d'être entourée des roches granitiques, calcaires et argileuse, source de grandes quantités de matières minérales. L'eau au cours de son trajet traverse les roches, ce qui peut drainer les substances minérales contenues dans ces roches. La forte teneur en calcium observée serait essentiellement liée à l'abondance des roches carbonatées dans la zone d'étude.

La dureté totale d'une eau est liée à sa teneur en sels de calcium et de magnésium. La dureté de l'eau influe essentiellement sur l'état des canalisations, sur les appareils de chauffage et sur le lavage du linge (Mahamane et Guel, 2015). Au vu des résultats de dureté totale obtenus, Il ressort que l'eau de Dassa est plus dure que celle de Cotonou. Ceci est en parfaite corrélation avec les teneurs élevées en magnésium et en calcium obtenues au niveau des échantillons d'eau de Dassa. Les concentrations obtenues au cours de cette étude ont été supérieures à celles mesurées par Coulibaly (2005) dans le cadre de ses investigations sur l'eau souterraine à Bamako. Le caractère très dur de l'eau de Dassa serait à la base du caractère peu moussant des eaux de cette localité.

Les résultats obtenus pour les bicarbonates sont similaires à ceux trouvés par Dovonon (2011) dans les eaux souterraines de Dassa. L'eau de Dassa s'est avérée très alcaline comparativement à celle de Cotonou. Les valeurs obtenues à Dassa ont été les mêmes que celles obtenues par Barhé et Bouaka (2013) au Congo Brazzaville avec l'eau de puits. En effet, les bicarbonates constituent l'essentiel de l'alcalinité de l'eau de Dassa. Ils peuvent provenir de la dissolution de formations carbonatées selon la réaction : $\mathrm{CaCO}_{3}+\mathrm{H}_{2} \mathrm{O} \rightarrow 2 \mathrm{HCO}_{3}^{-}+\mathrm{Ca}^{2+}$ (Mahamane et Guel, 2015).

L'iode est un élément minéral et un oligo-élément dont la carence entraîne des perturbations qui provoquent des lésions cérébrales, le crétinisme, et entraînent des fausses couches, une réduction de la fertilité et le goitre. Ces troubles constituent la cause d'arriération mentale la plus importante et aussi la plus facile à prévenir (Sezan et al., 2009). Les mesures des concentrations de l'iodure allant de $0,0 \mathrm{mg} / \mathrm{l}$ à $44 \mathrm{mg} / \mathrm{l}$ trouvées par Sezan et al. (2009) sur l'eau de puits sont plus élevées que celles obtenues dans la présente étude.

Le dosage des autres ions tels que sulfates, fluorures, fer total et chlorures a montré des valeurs presque négligeables. Les travaux de Coulibaly (2005) ont également montré que les valeurs obtenues sont presque nulles à comparer avec la valeur maximale admissible au Bénin. Les valeurs obtenues sont presque similaires à celles de Babadjide et al. (2011) et de Lagnika et al. (2014). La légère différence observée au niveau des résultats de ces paramètres serait certainement due au fait que l'eau de la SONEB était prétraitée comparativement à l'eau de pluies et de puits.

\section{Conclusion}

L'eau, principale source de vie dans les règnes végétal et animal, présente différentes qualités organoleptiques selon le milieu de vie. Les six (06) échantillons d'eau de consommation de Cotonou et de Dassa analysés sont tout à fait propres à la consommation des populations des deux localités et répondent à la norme admise. Des résultats des analyses physico-chimiques sur l'eau de boisson de Cotonou et de Dassa, il ressort que les paramètres évalués sont en forte proportion dans l'eau de Dassa que dans l'eau de Cotonou; en ce sens que l'eau de Dassa est plus concentrée en bicarbonates, magnésium, calcium, dureté totale et alcalinité. Ce qui pourrait expliquer le caractère moins moussant et le goût terne souvent remarqués au niveau de l'eau de Dassa comparativement à celle de Cotonou.

\section{REMERCIEMENTS}

Nos vifs remerciements à Monsieur AVOCANH Gautier, chef du Service QualitéEau de la Direction Général des Eaux au 
Bénin pour sa contribution technique aux analyses effectuées à la DG-Eau.

\section{REFERENCES}

Adejuwon JO, Mbuk CJ. 2011. Biological and physicochemical properties of shallow wells in Ikorodu town, Lagos Nigeria. Journal of Geology and Mining Research, 3(6): 161-168.

AFNOR (Association Française de Normalisation) 1986. Recueil des Normes Françaises. Eds Afnor : Paris.

Agbataou A. 2010. Evaluation et suivi du traitement de l'eau à l'usine de vêdoko, p. 57.

Akomagni A, Guidibi E. 2006. Monographie de la commune de Cotonou, p.47.

Awo-Affouda U. 2009. Projet d'AEP des villes de Savè, Dassa-zoumè, Glazoué, Savalou à partir des eaux de surface, p.97.

Babadjide C, Vissin E, Ouassa K, Adekambi A. 2011. Qualité d'eau consommée par la population et les maladies hydriques dans la commune de Kétou (Bénin), Université d'Abomey-Calavi, XXVI ${ }^{\text {èm }}$ colloque de l'Association Internationale de Climatologie, p. 81-86.

Barhé TA, Bouaka F. 2013. Caractérisation Physicochimique et Chloration des Eaux de Puits Consommées dans la Ville de Brazzaville-Congo. VJ. Mater. Environ. Sci., 4(5): 605-612.

Coulibaly K. 2005. Etude de la qualité physico-chimique de l'eau des puits de certains quartiers du district de Bamako, Thèse de doctorat, Université de Bamako, p. 69.

Coulibaly K. 2005. Etude de la qualité physico-chimique de l'eau des puits de certains quartiers du district de Bamako, Faculté de médecine de pharmacie, Thèse de doctorat d'état, Université de Bamako, p. 69.

Dégbey C, Makoutode M, Ouendo E-M, Fayomi B, De Brouwer C. 2008. La qualité de l'eau de puits dans la commune d'Abomey-Calavi au Bénin. Environnement, Risques et Santé, 7(4): 279-283. doi: 10.1684/ers.2008.0158
Derwich E, Benaabidate L, Zian A, Sadki O, Belghity D. 2010. Caractérisation physicochimique des eaux de la nappe alluviale du Haut Sebou en aval de sa confluence avec Oued Fès. Larhyss Journal, 08: 101-112.

DG-Eau 2007. Adduction d'Eau Villageoise, Guide à l'Usage des Communes. Direction Générale de l'Eau : Cotonou, Bénin ; 67.

Dovonon L. 2011. Qualité chimique des eaux souterraines dans la commune de Dassazoumé (Bénin): Impacts sanitaires des teneurs hors normes en fluorure et essais de traitement à l'os calciné de bovin, thèse de doctorat, FAST, Université d'Abomey-Calavi, p. 206.

Dovonon LFC, Soclo HH, Gbaguidi MAN, Youssao A. 2011. Utilisation des os calcinés dans la défluoruration des eaux contaminées : Détermination expérimentale de la température de calcination et de la granulométrie optimales des os. Int. J. Biol. Chem. Sci., 5(4): $\quad 1712-1726, \quad$ DOI : http://dx.doi.org/10.4314/ijbcs.v5i4.34

Festy B, Hartemann P, Ledrans M, Levallois P, Payment P, Tricard D. 2003. Qualité de l'eau, In : Environnement et santé publique - Fondements et pratiques, p.333-368.

Lagnika M, Ibikounle M, Montcho JC, Wotto VD, Sakiti NG. 2014. Caractéristiques physico-chimiques de l'eau des puits dans la commune de Pobè (Bénin, Afrique de l'ouest), J. Appl. Biosci., 79: 6887-6895. Doi: http://dx.doi.org/10.4314/jab.v75i1.12

Mahamane AA, Guel B. 2015. Caractérisations physico-chimiques des eaux souterraines de la localité de Yamtenga (Burkina Faso). Int. J. Biol. Chem. Sci., 9(1): 517-533. DOI : http://dx.doi.org/10.4314/ijbcs.v9i1.44

Makoutode M, Assani AK, Ouendo EM, Agueh VD, Diallo P. 1999. Qualité et mode de gestion de l'eau de puits en milieu rural au Bénin: cas de la souspréfecture de Grand-Popo. Médecine d'Afrique Noire, 46(11): 528-534. 
Odoulami L. 2009. La problématique de l'eau potable et la santé humaine dans la ville de Cotonou (République du Bénin), Thèse de Doctorat Unique, Université d'Abomey-Calavi, p.230.

OMS 1994. Directives de qualité pour l'Eau de boisson, vol 1, Deuxième édition, Genève.

Ould Cheikh MEK, El Kacemi K, Idrissi L. 2011. Caractérisation physico-chimique des eaux d'alimentation de la ville de Tijikja (Mauritanie). Int. J. Biol. Chem. Sci., 5(5): 2133-2139. DOI : http://dx.doi.org/10.4314/ijbcs.v5i5.33

Öztürk N, Bektaş TE. 2004. Nitrate removal from aqueous solution by adsorption onto various materials. Journal of Hazardous Materials, 112(1): 155-162.

Rodier J. 2009. L'Analyse de l'Eau (9ème édition). Ed. Dunod : Paris.

Sal M. 2010. Evaluation de la qualité microbiologique et physico-chimique de l'eau de l'adduction d'eau villageoise de Belefoungou-Centre (Djougou), p. 47.

Sezan A, Ahokpe M, Akpona S. 2009. Troubles dus aux carences iodées au Bénin. International Journal of Biological and Chemical Sciences, 3(6): 1246-1257.

Sintondji LO, Awoye HR, Agbossou KE. 2008. Modélisation du bilan hydrologique du bassin versant du Klouau CentreBénin: Contribution à la gestion durable des ressources en eau. Bulletin de la Recherche Agronomique du Bénin, 59: 35-48.

Tampo L, Ayah M, Kodom T, Tchakala I, Boguido P, Bawa L, Djaneye B. 2014. Impact de la demande en chlore et de la chloration sur la désinfection des eaux de puits des quartiers de Lomé : cas des quartiers de Démakpoé et d'Agbalépédogan (Togo). Journal of Applied Biosciences, 75: 6272-6281. Doi: http://dx.doi.org/10.4314/jab.v75i1.12 